\title{
Cognitive Performance as a Predictor of Functional Capacity in Schizophrenia
}

\author{
Leila Khabir \\ MA of Clinical Psychology, Lecturer, Dept. of Psychology, Payame Noor University \\ Email: Farsielham@yahoo.com.com
}

Fatemeh Farsi

BA in Clinical Psychologist, Department of Psychology, Islamic Azad University, Branch of Arsenjan, Iran

Maryam Hasir Baf

MA in Clinical Psychology, Department of Psychology, Islamic Azad University, Branch of Arsenjan, Iran

\section{Doi:10.5901/mjss.2015.v6n2s1p141}

\section{Abstract}

Introduction: Schizophrenia is a severe mental illness characterized by cognitive deficits as well as vocational impairment and interpersonal difficulties. This study examined the link between Continues Performance and everyday living skills. Methodes: Sample was 20 Schizophrenia (11 male and 9 female) selected using convenience sampling among individual with Schizophrenia diagnostic and 20 (10 male and 10 female) were matched with them as control group considering sex, age, marriage and education. Instruments were used in this study include: UCSD Performance-Based Skills Assessment Test (UPSA) and Continues Performance Test (CPT). Results: MANOVA showed a significant main effect for five domain of UPSA and four domain of UPSA. CPT predicted UPSA in schizophrenia. Conclusions: Individuals with schizophrenia would perform significantly worse in everyday living skills and on CPT. In fact poorer performance in CPT predicted basic skills performance in schizophrenia. This will help to determine what specifically needs to be targeted in rehabilitation of cognitive symptoms for those with schizophrenia, and that may reveal remediation in functional capacity as well.

Keywords: Schizophrenia, Continues Performance, everyday living skills

\section{Introduction}

Schizophrenia is a mental illness that is typified by debilitating symptoms of disordered thought, behavior, and emotions. The illness affects approximately $1 \%$ of the world's population and accounts for $1-2 \%$ of national healthcare costs in industrial countries $(1,2,3)$. Schizophrenia is a heterogeneous disorder in terms of symptoms, illness course, and etiology (4). This heterogeneity is expressed clinically in many ways. For example, patients vary in presence and severity of positive and/or negative symptoms. Positive symptoms include delusions (false beliefs) and hallucinations (false perception of sensory experience). Negative symptoms include a withdrawal of normal affect such as alogia (poverty of speech), anhedonia (inability to feel pleasure), alexithymia (inability to express emotion), as well as social withdrawal (5).Other clinically significant evidence for heterogeneity in this population are differences in the duration and amount of psychotic episodes, illness comorbidity, cognitive ability, and treatment response $(6,7)$. A diagnosis of schizophrenia is satisfied by meeting two or more of the following symptoms (or only one symptom in the case of a delusion of bizarre nature) that are present for the majority of the time during a one-month period): delusions, hallucinations, negative symptoms (e.g.,alogia, alexithymia, and/or blunted affect), disorganized or catatonic behavior, and disorganized speech. To meet criteria for schizophrenia, an individual must also have experienced pervasive disturbance of social or vocational functioning resulting from the symptoms lasting for at least six months. Symptoms must not be a product of a medical condition or substance use disorder or better classified by a pervasive developmental disorder (8). Schizophrenia is a severe mental illness characterized by cognitive deficits as well as vocational impairment and interpersonal difficulties. Individuals with schizophrenia also tend to have deficits in everyday living and limits in attention, focus, and vigilance. These difficulties contribute to the pervasiveness of the disorder, creating problems in job seeking, job maintenance, and activities of daily living (3).

Disruptions in independent living, social skills, and vocational functioning are characteristic of schizophrenia. 
Problems in self-care and maintenance contribute to decreased quality of life (general well-being and satisfaction with daily life and circumstance) and societal costs, such as the high cost of Medicare, Medicaid, and tax related hospital costs. Many of these deficits are pervasive, often begin in the early stages of the illness, and can be related to a great deal of the suffering that typify this disorder $(9,10,11,12)$. Functioning is a multi-dimensional concept. For individuals with schizophrenia, deficits in functioning occur in everyday living skills such as planning and keeping appointments, appropriate dressing, contacting appropriate people in emergency situations, medication management, and appropriate conversation with strangers $(9,11)$. These impairments also manifest in difficulty maintaining jobs or living independently. For example, approximately $62 \%$ of individuals with schizophrenia do not live independently (outside of supervised group housing or without relatives), and employment rates for those with schizophrenia are below $10 \%$ cross-culturally (13). To operationalize our definition of functioning in everyday living skills or capacity, we use a description of functioning that includes ability to appropriately address emergency situations, interact in social situations, focus on attending to personal needs in social situations, appointment keeping, and independent bill paying, as well as how to figure out transportation.We suggest that these are adaptive abilities needed for everyday living and interpersonal/social activities. Other definitions of functioning in schizophrenia include basic assessment of activities of daily living, such as problems with fundamental hygiene care (e.g., teeth brushing and showering) that prevent independent living and hospital discharge (14). For this study, we are more interested in functioning in everyday life skills, as there is a demonstrated relationship with independent living and quality of life (15), and a relationship between deficits in these skills and general cognitive deficits(16). In the laboratory, functioning in schizophrenia can be assessed by a number of different measures including tasks of everyday living. The University of California San Diego (UCSD) Performance-Based Skills AssessmentBrief Version (UPSA) is one example of such a measure. The UPSA was specifically designed for the assessment of individuals with schizophrenia. This particular measure, used in this study, is a 30 minute evaluation of performance in everyday living skills, like those mentioned above, and is credited for being tolerable, valid, reliable, and cross-culturally sound $(17,18)$. Evidences suggest impairments in functioning for individuals with schizophrenia, and these impairments seem to be pervasive throughout the course of the illness (19). The specific domains evaluated by the UPSA are pertinent to this project, as the measure elicits specific relationships between cognitive performances and deficits in everyday living skills (16).

Cognitive functioning refers to how individuals perform in cognitive domains such as memory (e.g., visual, spatial, working), executive functioning, and attention (19). Individuals with schizophrenia show impairments in nearly every domain of cognition (20).Cognitive functioning can be evaluated comprehensively to determine general intelligence ability. Cognitive domains of memory and attention can also be assessed differentially. Individuals with schizophrenia show impairments in nearly every domain of cognition (19). Researchers have shown that patients with schizophrenia tend to score in the lowest 5-10\% of the general population on cognitive assessments (21). Furthermore, at-risk and firstepisode studies, representing individuals across the schizophrenia-spectrum, indicate that deficits are present at onset, and even prior to onset of schizophrenia symptoms $(22,23,24)$. Researchers have shown that patients with schizophrenia tend to score in the lowest $5-10 \%$ of the general population on cognitive assessments (25). A metaanalysis by Heinrichs and Zakzanis (1998) considered the cognitive performance of 7,420 individuals (204 studies) with schizophrenia who demonstrated a consistent pattern of marked disturbance across attention, executive functioning, visuospatial ability, memory and language (21). Some studies showed Individuals with schizophrenia differed from Individuals with depression and control group in Continues Performance $(26,27,28)$.

Despite major advances in our understanding of cognitive limitations in schizophrenia, little is known as to how cognitive functioning relates to the pathophysiology of the illness and how exactly it underlies other difficulties associated with the disease (e.g., everyday living).Some researchers propose that that there is a link between cognitive impairments and functional impairments. For example, the literature has demonstrated positive correlations between deficits in domains of basic living and a wide range of cognitive impairments, such as executive functioning (29), verbal memory (30), attention (31), and working memory (32), to name a few. Interestingly, researchers have suggested that everyday living skills and cognitive symptoms are both pervasive from before diagnosis and through the lifespan. For those with schizophrenia, it is reported that attention, problem solving, and reasoning are most important in comparison to other cognitive domains in predicting performance in vocational-related tasks (33).

The basis of the predictive relationship between cognitive performance and everyday living skills is a critical knowledge gap regarding our understanding of the link between these two important aspects of functioning. The research discussed above demonstrates a number of different theories to connect this possible link. Cognitive functioning is complicated and multifaceted, and there is little consensus on which specific cognitive substrata may underlie deficits in everyday living skills.

It has demonstrated positive correlations between deficits in domains of basic living and a wide range of cognitive 
impairments, such as executive functioning (29), verbal memory (30), attention (31), and working memory (32). Oie, Sundet, and Ueland (2010) showed a relationship between baseline cognitive dysfunction and deficits in social functioning at a 13-year follow-up of those with early-onset schizophrenia (34). In this study Continues Performance was considered as a Cognitive functioning that assessed attention permanence.

This study examined the link between Continues Performance and everyday living skills. A primary goal of this study was to replicate previous findings that individuals with schizophrenia struggle with everyday living skills compared to non-psychiatric controls. Another study aim was to determine whether individuals with schizophrenia demonstrate exacerbating Continues Performance cognitive deficits compared to controls. Additionally, the objective was to evaluate whether Continues Performance are predicted everyday living skill deficits in individuals with schizophrenia.

\section{Methods}

The study design was correlational. Sample was 20 Schizophrenia (11 male and 9 female) selected using convenience sampling among individual with Schizophrenia diagnostic and 20 (10 male and 10 female) were matched with them as control group considering sex, age, marriage and education .

Instruments were used in this study include:

UCSD Performance-Based Skills Assessment Test (UPSA): The UPSA is the brief version of the UCSD performance-based skills assessment test and is a performance measure of a person's everyday living skills in five domains of daily living: 1)Financial Skills, 2)Communication, 3)Organization/Planning, 4)Transportation, and 5)Household Management. The UPSA takes approximately 30 minutes to administer and has been shown to have high test-retest reliability and participant tolerability above and beyond other co-primary measures. Possible range of scores for each subscale is between $0-20$ and for total score is between $0-100$. This instrument was translated and validated (35). In this study UPSA was translated and validated.

Continues Performance Test (CPT): The CPT was designed in 1956. The aim of CPT is assessment inattentiveness, impulsivity, sustained attention and vigilance. The CPT respondents are required to press the space bar or click the mouse whenever any letter except the letter ' $X$ ' appears on the computer screen. The following types of measures are provided by the CPT program: 1) Errors Commissions: Incorrect responses to non-targets, 2) Errors Omissions: Missed targets by block, 3)Reaction Time: Response speed, 4)Matches Successfully Recognized: Ability to discriminate between targets (non-X) and non-targets (X) (36). Reliability And validity of CPT were reported suitable (37).

\section{Results}

Demographic data for participants in the schizophrenia and control group were showed (Table 1,2). Range of age in schizophrenia and control group was 18-49 (33.23 \pm 8.93$)$ and 23-53 (35.26 \pm 9.51$)$.

Table 1. Demographic data (Sex and Marriage) for participants in the schizophrenia and control group

\begin{tabular}{lcccccccc}
\hline & \multicolumn{4}{c}{ Sex } & \multicolumn{4}{c}{ Marriage } \\
\cline { 2 - 10 } Group & \multicolumn{3}{c}{ Male } & \multicolumn{2}{c}{ Female } & \multicolumn{2}{c}{ Single } & \multicolumn{3}{c}{ Married } \\
& Frequency & Percent & Frequency & Percent & Frequency & Percent & Frequency & Percent \\
Schizophrenia & 11 & 55 & 9 & 45 & 7 & 35 & 13 & 65 \\
Control & 10 & 50 & 10 & 50 & 10 & 50 & 10 & 50 \\
\hline
\end{tabular}

Table 2. Demographic data (Education) for participants in the schizophrenia and control group

\begin{tabular}{lcccccc}
\hline \multirow{2}{*}{ Group } & \multicolumn{6}{c}{ Education } \\
\cline { 2 - 7 } & \multicolumn{2}{c}{ Under Diploma } & \multicolumn{2}{c}{ Diploma } & \multicolumn{2}{c}{ MB ad more } \\
Schizophrenia & Frequency & Percent & Frequency & Percent & Frequency & Percent \\
Control & 7 & 35 & 8 & 40 & 5 & 25 \\
\hline
\end{tabular}

Group comparisons was conducted to determine if there were demographic differences (sex, age, marriage and education) between the control group and the schizophrenia group in order to account for any confounding variables that could impact later analyses. No significant differences in $\operatorname{sex}\left(X^{2}=0.21, P=0.7\right)$, education $\left(X^{2}=0.50, P=0.6\right)$, marriage $\left(X^{2}\right.$ 
$=0.01, P=0.9)$ and age $(F=0.29, P=0.8)$ were found using chi square analysis for sex, education and marriage and ANOVA for age.

Levene Test was not significant in four subscales of CPT and Pillai's Trace was significant ( $F=6.03, P=0.0001)$. There would be a main effect for group for cognitive performance, the schizophrenia group would perform significantly worse on CPT. A MANOVA showed a significant main effect for our domain of CPT (Table 3).

Table 3. Comparison of CPT in the schizophrenia and control group

\begin{tabular}{lcccccc}
\hline \multicolumn{1}{c}{ Scource } & & Sum of Square & df & Mean Square & F & $\boldsymbol{P}$ \\
\hline \multirow{3}{*}{ Errors Commissions } & Group & 575.49 & 1 & 575.49 & 26.40 & 0.0001 \\
& Error & 2397.78 & 77 & 2397.78 & & \\
& Total & 29603 & 79 & & & \\
\multirow{3}{*}{ Errors Omissions } & Group & 602.60 & 1 & 602.60 & 31 & 0.0001 \\
& Error & 2137.64 & 77 & 19.43 & & \\
\multirow{3}{*}{ Reaction Time } & Total & 157106 & 79 & & & \\
& Group & 2356.85 & 1 & 2356.85 & 37.08 & 0.0001 \\
\hline \multirow{3}{*}{ Matches Successfully Recognized } & Error & 6990.56 & 77 & 63.55 & & \\
& Total & 667403 & 79 & & & \\
& Group & $2.04 \mathrm{E}$ & 1 & $2.04 \mathrm{E}$ & $1.37 \mathrm{E}$ & 0.0001 \\
& Error & $1.03 \mathrm{E}$ & 77 & 148948.87 & & \\
\hline & Total & $2.40 \mathrm{E}$ & 79 & & & \\
\hline
\end{tabular}

Levene Test was not significant in five subscales of UPSA and Pillai's Trace was significant ( $F=450.49, P=0.0001)$. There would be a main effect for group for cognitive performance, the schizophrenia group would perform significantly worse on UPSA. A MANOVA showed a significant main effect for five domain of UPSA. In fact individuals with schizophrenia would perform worse in everyday living skills (Table 4).

Table 4. Comparison of UPSA in the schizophrenia and control group

\begin{tabular}{|c|c|c|c|c|c|c|}
\hline Scource & & Sum of Square & df & Mean Square & $F$ & $P$ \\
\hline \multirow{3}{*}{ Financial Skills } & Group & 1254.40 & 1 & 1254.40 & 109.05 & 0.0001 \\
\hline & Error & 437.10 & 38 & 11.50 & & \\
\hline & Total & 6754 & 40 & & & \\
\hline \multirow{3}{*}{ Communication } & Group & 1144.90 & 1 & 144.90 & 96.46 & 0.0001 \\
\hline & Error & 451 & 38 & 11.86 & & \\
\hline & Total & 6932 & 40 & & & \\
\hline \multirow{3}{*}{ Organization/Planning } & Group & 1134.22 & 1 & 1134.22 & 151.46 & 0.0001 \\
\hline & Error & 284.55 & 38 & 7.48 & & \\
\hline & Total & 4713 & 40 & & & \\
\hline \multirow{3}{*}{ Transportation } & Group & 1071.22 & 1 & 1071.22 & 136.80 & 0.0001 \\
\hline & Error & 297.55 & 38 & 7.83 & & \\
\hline & Total & 7201 & 40 & & & \\
\hline \multirow{3}{*}{ Household Management } & Group & 1677.02 & 1 & 1677.02 & 216.50 & 0.0001 \\
\hline & Error & 294.35 & 38 & 7.74 & & \\
\hline & Total & 6487 & 40 & & & \\
\hline
\end{tabular}

The analysis with enter regression showed poorer performance in CPT predicted basic skills performance in schizophrenia (Table 5). $64 \%$ of variation basic skills performance in schizophrenia was predicted by Poorer performance in CPT (Table 6).

Table 5. Prediction UPSA rely on CPT

\begin{tabular}{cccccc}
\hline Model & Sum of Square & df & Mean Square & F & $\boldsymbol{P}$ \\
\hline Regression & 953.89 & 1 & 953.89 & 32.72 & 0.0001 \\
Residual & 524.65 & 18 & 29.14 & & \\
Total & 1478.55 & 19 & & & \\
\hline
\end{tabular}


Table 6. Regression Coefficients

\begin{tabular}{ccccccc}
\hline Step & $\mathbf{R}$ & $\mathbf{R}^{2}$ & $\mathbf{B}$ & $\boldsymbol{\beta}$ & $\mathbf{t}$ & $\boldsymbol{P}$ \\
\hline $\mathbf{1}$ & 0.80 & 0.64 & 0.95 & 0.80 & 5.72 & 000.1 \\
\hline
\end{tabular}

\section{Discussion}

The first aim of this study was to compare everyday living skills between controls and patients with schizophrenia. The results of this study indicated that individuals with schizophrenia performed worse in everyday living skills compared to healthy controls. This finding was supported by Addington and Addington (1999), Bowie, Reichenberg, Patterson, Heaton, and Harvey (2006), Galuppi, Turola, Nanni, Mazzoni, and Grassi (2010), Green (1996), Harvey, Helldin, Bowie, Heaton, Olsson, Hjarthag, Patterson (2009), Ikebuchi, Satoh and Anzai (2008), Marwaha, Johnson, Bebbington, Angermeyer, Brugha, Toumi (2007), Patterson, Goldman, McKibbin, Hughs and Jeste (2001), Pitknen, Vlimki, Kuosmanen, Katajisto, Koivunen, Htonenm, Knapp (2010) and Simon (2003) that showed those with schizophrenia suffer from severe disruptions in the capacity to carryout everyday living skills.

The second aim was to evaluate whether the schizophrenia group would perform significantly worse on CPT. Those with schizophrenia performed worse in CPT compared to non-psychiatric controls. This finding was supported by Albus Hubmann, Mohr, Hecht, Hinterberger-Weber, Seitz, and Kuchenhoff (2006), Asarnow (1999), Cornblatt, Lenzanweger and Erlenmeyer-Kimiling (1989), Keefe, Eesley, and Poe (2007), Hasani and Hadianfard (2003), Heinrichs and Zakzanis (1998), Keefe, Eesley, and Poe (2007), Lezak (1994), Liu , Chiu and Chang (2002) and Ragland (2003).

The last was to evaluate whether Continues Performance are predicted everyday living skill deficits in individuals with schizophrenia. The results indicated that CPT could predicted everyday living skill deficits. This finding was supported by Erlenmeyer-Kimling, Rock, Roberts, Janal, Kestenbaum, Cornblatt (2007), Fujii, Wylie and Nathan (2004), Oie, Sundet, and Ueland (2010), Puig, Penade, Gasto, Catalan, Torres, Salamero (2008), Ritsner (2007) and Tan (2009).

In generally, individuals with schizophrenia performed worse in UPSA and CPT compare to control group.

\section{Conclusion}

Ths results showed low performance in CPT predicted worse performance in UPSA. This will help to determine what specifically needs to be targeted in rehabilitation of cognitive symptoms for those with schizophrenia, and that may reveal remediation in functional capacity as well.

\section{References}

Hu TW. Perspectives: An international review of the national cost estimates of mental illness. J Mental Health Policy Economics 2006; 9(1): 13-3.

Tsuang MT, Stone WS, Faraone SV. Schizophrenia: A review of genetic studies. Harvard Review of Psychiatry 1999; 7(4): 207-185.

Wu EQ, Birnbaum HG, Shi L, Ball DE, Kessler RC, Moulis M, Aggarwal J. The economic burden of schizophrenia in the United States in 2002. J Clinical Psychiatry 2005; 66(9): 1129-1122.

Cohen AS, Docherty NM. Symptom-oriented versus syndrome approaches to resolving heterogeneity of neuropsychological functioning in schizophrenia. J Neuropsychiatry Clinical Neuroscience 2005; 17(3): 390-384.

Adams HE, Sucker BE. Comprehensive handbook of psychopathology(3rd Ed.).New York: Springer. 2001.

Bruder GE, Alschuler DM, Kroppmann CJ, Fekri S, Gil RB, Jarskog LF, Wexler BE, et al. Heterogeneity of auditory verbal working memory in schizophrenia. J Abnormal Psychology, 120(1): 97-88.

Case M, Stauffer, Ascher-Svanum H, Conley R, Kapur S, Kane JM, . . Kinon, BJ. The heterogeneity of antipsychotic response in the treatment of schizophrenia. J Psychological Medicine 2010; 10-1.

American Psychiatric Association. Diagnostic and statistical manual of mental disorders (4th Ed.). Washington, DC: American Psychiatric Association 1994.

Addington J, Addington D. Neurocognitive and social functioning in schizophrenia. Schizophrenia Bulletin 1999; 25(1): $182-173$.

Bowie CR, Reichenberg A, Patterson TL, Heaton RK, Harvey PD. Determinants of real-world functional performance in schizophrenia subjects: Correlations with cognition, functional capacity, and symptoms. American J Psychiatry 2006; 163(3): 425-418.

Galuppi A, Turola MC, Nanni MG, Mazzoni P, Grassi L. Schizophrenia and quality of life: How important are symptoms and functioning? International J Mental Health Systems2010; 4(1): 31.

Simon GE. (2003). Social and economic burden of mood disorders. Biological Psychiatry 2003; 54(3): 208-215.

Marwha S, Johnson S, Bebbington P, Stafford M, Angermeyer MC, Brugha T, . . Toumi M. Rates and correlates of employment in people with schizophrenia in the UK, France and Germany. The British J of Psychiatry 2007; 191: 37-30. 
Ikebuchi E, Satoh S, Anzai N. What impedes discharge suppoor persons with schizophrenia in psychiatric hospitals? Seishin Shinkeigaku Zasshi 2008; 110(11): 1022-1007.

Pitknen A, Vlimki M, Kuosmanen L, Katajisto J, Koivunen M, Htonen $\mathrm{H}$, . . . Knapp M. Patient education methods to support quality of life and functional ability among patients with schizophrenia: A randomized clinical trial. Quality of Life Research 2010; 21(2): 247256.

Green MF. What are the functional consequences of neurocognitive deficits in schizophrenia? American Journal of Psychiatry 1996; 153(3): 330-321.

Harvey PD, Helldin L, Bowie CR, Heaton RK, Olsson AK, Hjarthag F, . . Patterson TL. Performance-based measurement of functional disability in schizophrenia: A cross-national study in the United States and Sweden. American J of Psychiatry 2009; 166(7): 827821.

Patterson T, Goldman S, McKibbin CL, Hughs T, Jeste DV. UCSD Performance-Based Skills Assessment: Development of a new measure of everyday functioning for severely mentally ill adults. Schizophrenia Bulletin 2001; 27(2): 245-235.

Lezak MD. Domains of behavior from a neuropsychological perspective: The whole story. Nebraska Symposium on Motivation 1994; 41 : 55-23.

Heinrichs RW, Zakzanis KK. Neurocognitive deficit in schizophrenia: A quantitative review of the evidence. Neuropsychology 1998; 12: 445-426.

Keefe RS. Cognitive deficits in patients with schizophrenia: Effects and treatment. J of Clinical Psychiatry 2007; 68 (14): $13-8$.

Albus M, Hubmann W, Mohr F, Hecht S, Hinterberger-Weber P, Seitz N, Kuchenhoff H. Neurocognitive functioning in patients with first episode schizophrenia :results of a prospective 5-year follow-up study. European Archives of Psychiatry Clinical Neuroscience 2006; 256(7): 451-442.

Asarnow RF. Neurocognitive impairments in schizophrenia: a piece of the epigenetic puzzle. European Child Adolescent Psychiatry 1999; 8 (1): 15-8.

Ragland, JD. Profiles of neuropsychologic function in schizophrenia. Current Psychiatry Report 2003; 5(4): 302-299.

Keefe RS, Eesley CE, Poe MP. Defining a cognitive function decrement in schizophrenia. Biological Psychiatry 2005; 57(6): 91-688.

Liu SK, Chiu CH , Chang CJ. Defiction sustained attention in schizophrenia and affective disorders: Stable versus statedep endent markers. American J Psychiatry 2002, 159: 982-975.

Cornblatt BA, Lenzanweger MF, Erlenmeyer-Kimiling L. The continuous performance test, identical paris version: II contrasting attention profiles in schizophrenia and depressed patients. Psychiatry Research 1989; 29: 86-65.

Hasani J, Hadianfard $\mathrm{H}$. The relationship between positive and negative symptoms of schizophrenia with attention permanence. Advances in Cognitive Science 2003; 5(3): 33-24.

Ritsner MS. Predicting quality of life impairment in chronic schizophrenia from cognitive variables. Quality of Life Research 2007, 16(6): 937-929.

Puig O, Penade, R, Gasto C, Catalan R, Torres A, Salamero M. Verbal memory, negative symptomatology and prediction of psychosocial functioning in schizophrenia. Psychiatry Research 2008; 158(1): 17-11.

Erlenmeyer-Kimling L, Rock D, Roberts SA, Janal M, Kestenbaum C, Cornblatt B, et al. Attention, memory, and motor skills as childhood predictors of schizophrenia-related psychoses: The New York High-Risk Project. American Journal of Psychiatry 2000, 157(9): $1422-1416$.

Fujii DE, Wylie AM., Nathan JH. (2004). Neurocognition and long-term prediction of quality of life in outpatients with severe and persistent mental illness. Schizophrenia Research 2004; 69(1): 73-67.

Tan, B. L. Profile of cognitive problems in schizophrenia and implications for vocational functioning. Australian Occupuational Therapy Journal 2009; 56(4), 228-220.

Oie M, Sundet K, Ueland T. Neurocognition and functional outcome inearly onset schizophrenia and attention-deficit/hyperactivity disorder: A 13-yearfollowup. Neuropsychology 2010; 25(1):35-25.

Harvey PD, Green MF, Nuechterlein KH. Latest developments in the matrics process. Psychiatry (Edgmont) 2010; 7(6): $52-49$.

Mahmood Aliloo M, Hamidi S and Shiravani A.Executive function, Sustained attention, Obsessive-compulsive symptoms, High schizotypy, Overlapping symptom. J Behavioral Sciences 2011; 9(3): 220-216.

Hadianfard H, Najarian B, Shokrkon $\mathrm{H}$ and Mehrabizadeh Honarmand M. Making the Persian form of the continuous performance tes. Psychology J 2000; 4(2): 440-338. 